\title{
Designing Interactive Pill Reminders for Older Adults: A Formative Study
}

\author{
Sepideh Ansari \\ 535 W Michigan, Indianapolis, IN, 46236, USA \\ seansari@iupui.edu
}

\begin{abstract}
Introduction: The real challenge for information technologies is not content or curriculum development, but the development of interactive mechanisms by which to make information individually relevant, timely, and, tailored to promote information sharing. At first, this article reviews common technological approaches accessible to elderly that are intended to increase adherence to medication. Then, in a formative process the study proposes a novel framework to design and evaluate an interactive automated pill reminder for older adults. The proposed interactive pill reminder offers a solution by dispensing medication to the elderly in a way that is secure, routine, and monitored by their physician or pharmacist. Considering the interaction tasks between the device and a physician or pharmacist, coupled with their work schedules, the device is designed so that it is simple enough for the industry to absorb it.
\end{abstract}

Background: Non-adherence among patients has been identified as a major public health problem that imposes a considerable financial burden upon modern health care systems. This burden includes $10 \%$ of hospital admissions, $23 \%$ of admissions to nursing homes and has been estimated to cost $\$ 100$ billion each year in the US. Information technology has been used both to measure and enhance adherence. Measurement has been through advanced technologies, such as smart pill-bottle caps, to capture medication-taking behavior and using information technology to collect and synchronize both frequency and time of opening of the medication bottle, with a central database.

Methodology: The development of the pill reminder includes three main stages: (1) Designing the high fidelity prototype based on heuristics and available guidelines and collecting necessary information applicable in the design of the interactive pill reminder (i.e., user requirement analysis, general requirements of small screen devices, and design principles for older adults); (2) Pilot testing the mid-fidelity interactive pill reminder prototype and analyzing the results (i.e., examining the mid-fidelity product by user interviews, a focus group, and a usability questionnaire); and (3) Proposing a list of tasks to improve the future high-fidelity prototype (i.e., proposing future high-fidelity design heuristics).

Conclusion: Non-adherence to medication is a major health burden specifically in the elderly population. Designing new automatic pill reminders for elderly can open new ways to improve adherence rates among them; however, more formative research needs to be conducted to establish preliminary design guidelines in designing such reminders. This study collected necessary information applicable in the design of the interactive pill reminder. The result was the development of a high fidelity prototype for an automatic pill reminder. 


\section{Introduction}

Non-adherence among patients has been identified as a major public health problem that imposes a considerable financial burden upon modern health care systems [15]. This burden includes $10 \%$ of hospital admissions, $23 \%$ of admissions to nursing homes [21] and has been estimated to cost \$100 billion each year in the US [15].

Context: Adherence among older adults should be increased. This can be applied by integrating an interactive design into a pocket size pill dispenser. Furthermore, pill dispensers should be secured (e.g., no access by children). Pill dispensers should also dispense only the pills that are required for a specific dose intake. An ideal interactive device should manage the reminder and the dispenser systems simultaneously.

Use: Interactive pill reminders should focus on this problem and offer a solution by dispensing medication to the elderly in a way that is secure, routine, and monitored by their physician or pharmacist. Considering the interaction tasks between the device and a physician or pharmacist, coupled with their work schedules, the device should also be designed so that it is simple enough for the industry to absorb it.

People: Primary users will be older adult patients that are required to take a long list of medications (e.g., complex diseases) for an extended period of time (e.g., chronic patients). Secondary users will be doctors and pharmacists who could upload and maintain the data necessary to continue the effectiveness of the device; however, these users will not be studied in the scope of this proposal.

Importance of Product: Lack of complete adherence to the medical regimen transcends the boundaries of disease categories and age groups [20]. Without intervention, adherence rates to long-term medication in high income countries are approximately $50 \%$ [18], while adherence in low and middle income countries may be even lower [23]. Millions of people don't take their medication correctly [15]. The consequence of mistaking medication can be very dangerous or even deadly [9]. Many people forgot to take their medications regularly, which can complicate their medical problems and raise the chance of unforeseen hospitalized and clinical trial. Thus, non-adherence to medication is a crucial health problem. An effective automated pill reminder can reduce the aforementioned risks and problems and thus save billions of dollars for patients, insurance companies and the government.

This paper introduces a new conceptual design for an interactive automated pill reminder specifically designed to appeal the older adult population. The usability of the mid-fidelity pill reminder design will be further validated by various methods such as interviews, focus groups, questionnaires and a user evaluation study.

\section{Background}

\subsection{Adherence to Medication}

The word "adherence" is preferred to compliance by many healthcare providers, because "compliance" suggests that the patient is passively following the doctor's orders and that the treatment plan is not based on a therapeutic alliance or contract established between the patient and the physician [15]. Indeed, adherence is a more 
neutral term than compliance, which can be construed as being judgmental. Despite these differences, both terms are commonly used as descriptions of medication-taking behavior [15].

Winnick (2005) defines adherence as: "the extent to which a person's behavior coincides with medical or health advice" ([22], p. e718). The term compliance, defined as 'to consent' or 'to do as asked', has been used instead of adherence for many years [6]. Other definitions of adherence add components such as knowledge of medication, timely filling of prescriptions, exact dosage, accurate timing of the doses, approximate sequence of taking the drug, correct length of therapy, and on-time attendance for follow-up appointments [20].

Treatment adherence rates are typically lower among older adults, mental disorders, and patients with chronic conditions as compared to those with acute conditions [11]. Persistence with medical treatment regimens among patients with chronic conditions and elderly is also disappointingly low [12].

\subsection{Importance of Adherence to Medication}

The problem of non-adherence with medication is not new. Hippocrates advised physicians to "keep watch also on the fault of patients which often make them lie about the taking of things prescribed" [13]. In an extensive review of medication adherence research, it was found that approximately $50 \%$ of patients do not take prescribed medications in accordance with physicians' instruction [20]. Poor adherence is to be expected in 30-50\% of all patients, irrespective of disease, prognosis or setting [14].

Medical non-adherence has also been identified as a major public health problem that imposes a considerable financial burden upon modern health care systems [14]. This burden has been estimated to cost $\$ 100$ billion each year in the USA [15], including being responsible for $10 \%$ of hospital admissions and $23 \%$ of admissions to nursing homes [21]. Poor adherence to medication regimens accounts for substantial worsening of disease, death, and increased health care costs in the United States [15]. Increased health care utilization and costs are common outcomes of failure to adhere to treatment regimens [17].

\subsection{Measuring Medication Adherence}

There is no gold standard method to measure adherence [2]. Fielding (1999) argues that although adherence and health status are linked there have been few empirical tests of this association. Studies of adherence frequently confound these concepts, using measures of health status and adherence interchangeably [10].

Vermeire [21] expands on the relationship between health outcome and adherence to treatment and categorizes adherence measurement into process-oriented and outcome-oriented. In the process-oriented approach, medication-taking behavior is the center of measurement while in the outcome-oriented approach the clinical outcomes of adherence to treatment are measured. For example, studies measuring process-oriented adherence are concerned with the number of doses not taken or taken incorrectly that jeopardize the therapeutic outcome. On the other hand, studies that measure outcome-orientated adherence emphasize the end-result or outcome of the actions taken. 
Rates of (process-oriented) adherence for individual patients are usually reported as the percentage of the prescribed doses of medication actually taken by the patient over a specified period. Some investigators have further refined the definition of adherence to include data on dose taking (i.e. taking the prescribed number of pills each day) and the timing of doses (i.e. taking pills within a prescribed period). This study of the interactive pill reminder for older adults will implement a combination of the dose taking and timing of doses in the adherence rate calculation.

\subsection{Barriers to Adherence}

Adherence research has focused on the extent and determinants of non-adherence, and strategies to improve adherence to treatment. Major predictors of poor adherence to medication include [15]: Presence of psychological problems; Presence of cognitive impairment; Treatment of asymptomatic disease; Inadequate follow-up or discharge planning; Side effects of medication; Patient's lack of belief in benefit of treatment; Patient's lack of insight into the illness; Poor provider-patient relationship; Presence of barriers to care or medications; Missed appointments; Complexity of treatment; and Cost of medication, copayment, or both. Claxton has argued that adherence is inversely proportional to frequency of dose [5] and thus considered the frequency of dose as a predictor of adherence rate.

Donovan [8] indicates that one of the major reasons for the lack of progress in compliance research is the absence of patient's perspective. Vermeire (2001) argues that barriers to adherence that are under the patient's control should attract more attention in research. More than 200 variables have been studied since 1975, but none of them can be considered as consistently predicting adherence: neither socioeconomic nor pathology-related factors [21]. Research into a phenomenon as complex as adherence is inevitably fragmented, because of the absence of a model or theory to integrate the different studies [21].

\subsection{Sample IT Applications in Medication Adherence}

"Patients frequently cite forgetfulness as the most common reason for not taking medication. Therefore, interventions that improve memory are likely to enhance patient adherence. Several cost-effective strategies are available that may help patients remember to take their medication. Patients should be encouraged to develop a home dosing routine for their medication use - a routine that enables a patient to take their medication at the same time each day and that involves cues that prompt medicationtaking rituals and enhance memory. Health care practitioners should encourage the use of medication adherence aids, such as calendars, pillboxes with built-in timer alarms, dosage counters, multi-alarm wrist watches, pagers with alarm and text reminders, medication diaries, phone reminders, computerized medication schedulers, and other adherence tools" [3].

Medication Reminder Software: Although various factors have been related to adherence, patient understanding of the illness and influence of treatment on it can affect treatment adherence. If information provided to the patients is individually tailored, it has a greater impact on patient behavior than providing generic information. In one such study on medication adherence in elderly patients with memory disorders, a 
PC-based software application to provide individually-tailored medication information was developed. Programmed in Visual Basic, the application automates a critical part of an intervention that includes assessment of patient health care literacy, preferred language, and information needs. The application provides printed output that includes the patient's name and answers to specific questions endorsed by the patient. Output is provided at one of two literacy levels and in Spanish as well as English. Patient adherence to cholinesterase inhibitor medications prescribed for memory problems was assessed longitudinally with an electronic recording device. Preliminary data collected from the research study showed that the device had a high degree of patient acceptability. The data also indicated that the use of the tailored information device was associated with high levels of sustained medication adherence [16].
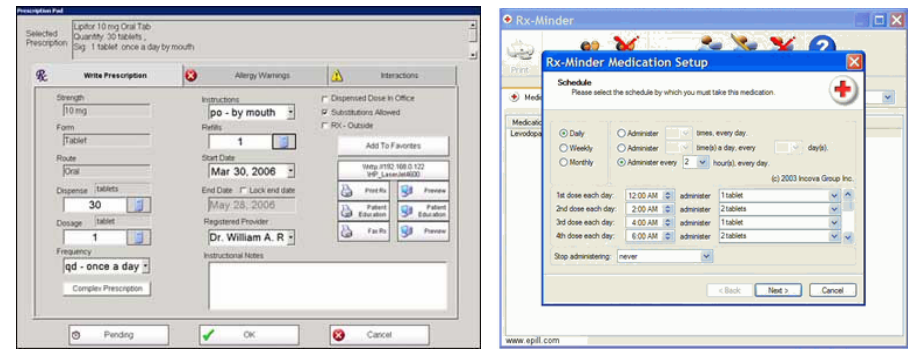

Fig. 1. PC-based Medication Reminder Software

Mobile Phone - SMS Reminders: Research has been done on wireless text messaging or SMS in improving people's adherence. It was found that innovative mobile healthcare solutions, based on portable devices like cell phones, may address some non-adherence aspects by helping outpatients to follow treatments agreed with their health providers [4]. A mobile application 'UbiMeds' has been developed to improve accessibility and support medication adherence for aging and disabled population [19]. In another pilot research study, customized messages were programmed into the user's mobile phones before they left their doctor's offices. As a result, a simulated SMS would arrive on the patient's phone when it's time to take the medication. The application on the phone also allowed them to anchor a medication event to a lifestyle event, i.e. always taken with lunch, so if your lunch schedule changes the medication reminder can, too. When it was time for a medication event the patient's phone would ring, a message would appear on the screen. When answered it asks the patient to touch the mobile phone to the medication bottle. The bottle had an embedded RFID chip, which can transfer information to the phone. The phone would then ask the patient if " $\mathrm{X}$ " is the medication they were taking and once confirmed, proceeds to instruct the patient on how to take the medicine. Every time the patient interacts with the application it records the event and confirms each event for clinicians to review from a Web portal in real-time or at a later date. This process resulted in an increase in 96 percent adherence in the pilot study [7].

Medication Event Monitoring System (MEMS): The MEMS monitors are drug packages with integral electronic micro circuitry designed to compile the dosing 
histories of ambulatory patients' prescribed medications. Each monitor consists of a conventional medicine bottle fitted with a special closure that records the time and date of each opening and closing of the container through integrated microcircuitry. Monitors are designed to be used by one patient with one drug. A reader transfers the dosing history data from the MEMS monitor to a MS-Windows based computer [1].

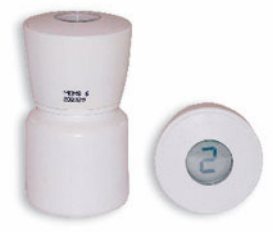

Fig. 2. Medication Event Monitoring System [1]

\section{Methods}

The design of an automatic pill reminder should go through multiple steps: (1) Collecting necessary information applicable in the design of the interactive pill reminder (i.e., user requirement analysis) and preliminary design of the pill reminder interface based on available guidelines and heuristics; (2) Pilot testing the mid-fidelity interactive pill reminder prototype and analyzing the results (i.e., examining mid-fidelity); and, (3) Proposing a list of tasks to improve the future high-fidelity prototype (i.e., proposing future high-fidelity). In the rest of this paper step 1, the preliminary design of the pill reminder interface through guidelines and heuristics, is explained. This is an ongoing research project and results of each step will be published separately.

\subsection{Usability and Experience Goals of the Users}

Based on the general requirements of small screen devices and design principles for older adults the following primary usability goals for users of the interactive pill reminder are developed:

\section{Usability}

-Effective - The Interactive pill reminder should aid the user by helping them load and consume the medications in exact amounts at consistent times each day. It will also have a calendar to help organize medications.

-Efficient - Loading medications should be simplified

- Easy to Learn - Using a touch screen, buttons must be clearly defined and intuitive. Appropriate feedback should be given for each action.

- Options/Flexibility - Color scheme, time, and the calendar should all be customizable to suit each user's preferences

- Safety-The user's personal and medical information should be stored in such a way that it is accessible only by the user (though with some exception to their primary health care physician, i.e. new/old prescription data, etc). And their medication must be kept safe in a secure sterilized compartment 


\section{Experience:}

-Enjoyability - The user will be able to be more independent and have a greater quality of life

- Helpful - The personal calendar will allow the user to take command of not only their medications but personal life as well.

- Motivating - The Interactive pill reminder will inspire users to take their medications correctly

- Inviting - The Interactive pill reminder should be aesthetically pleasing, inviting the user to pick it up and interact with it through clear and clean menus, as well as right-brained icons and pictures.

\subsection{User Requirement Analysis}

Based on the literature review conducted on adherence to medication and a review of current pill reminders the following objectives and interactions are considered for the initial design of the interactive pill reminder:

\section{Loading Medication}

- Use a barcode scanner, similar to ones already used by Pharmacists to organize data - all relevant data will be uploaded to the interactive pill reminder database

- Interactive pill reminder will collection the following information:

- Which time each day he/she would like to take medication

- How many pills he/she must place in each compartment

- Compartments for each day of the week will possess LED's:

○ Container empty - no flash or highlight

- LED on (but not flashing) - medication loaded

- Taking medication

○ Various alarms will go off

- Vibration, aural, visual

- Fingerprint will be scanned to ensure security

- Before user is prompted to take medication, the screen will display:

- The name, picture, dosage, and physical description of the medication

- Display a button for a description of what the medication treats, as well as the information pertaining to the physician who prescribed the medicine

- User will take medications, corresponding compartmental LED's will flash

- As the medication is taken, the touch screen will prompt the user with a question, asking them if they have taken the medication

-If "yes," compartments will stop flashing

-If answer is "no" or user forgets specific interval - screen will flash, the alarm will sound at a higher pace, and the device itself will vibrate, as to alert the user 
- Medication Database

- Will have all known medications for reference

- New medications can be added through Dr or Pharmacist via USB

- Miscellaneous

- Possess a personal calendar - remind the user of personal dates (birthdays, etc), doctor appointments, and medication schedule

- Wake-up alarm clock

o Be small enough to fit in pocket

- Be rechargeable

The initial list of aforementioned requirements (usability and user requirements) will be modified and updated based on the outcomes of the rest of the user requirement analyses to be performed in future steps.

\section{Proposed High Fidelity Prototype}

Based on the developed design guidelines based on the available guidelines for elderly the following high fidelity prototype is designed:

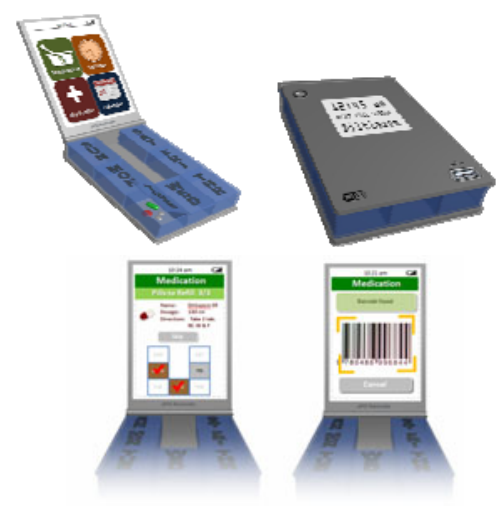

Fig. 3. Sample screenshots of the proposed prototype

\section{Future Work}

A user study is planned to complete steps 2 and 3 of the methodology:

User Interviews: 10 participants (both male and female) more than or equal to $65 \mathrm{yrs}$ of age based on a convenient sample selection will be enrolled in a face-to-face interview. The interview will be based on semi-open questions.

Focus Group: 8 participants (both male and female) more than or equal to 65 yrs of age based on a convenient sample selection will be invited in a face-to-face focus group. Open ended discussion topics will be presented by the focus group leader. 
Questionnaire: 20 participants (both male and female) more than or equal to 65 yrs of age based on a convenient sample selection will be enrolled in a mailed questionnaire. The questionnaire will mainly consist of Likert-scaled questions examining the usability of proposed design elements in the interactive pill reminder. The questionnaire will include open-ended questions to include any remaining issues.

User Interviews, Focus Group and Open Ended Questions of the Questionnaire: Grounded theory will be used to generate new ideas and a possible a hypothesis.

Likert-Scaled Questions of the Questionnaire: SPSS statistical package will be used to calculate the averages and possible significant differences in the facilitators and barriers to the adoption of the proposed interactive pill reminder

\section{Conclusion}

Non-adherence to medication is a major health burden specifically in the elderly population. Designing new automatic pill reminders for elderly can open new ways to improve adherence rates among them; however, more formative research needs to be conducted to establish preliminary design guidelines in designing such reminders. This study collected necessary information applicable in the design of the interactive pill reminder. The result was the development of a high fidelity prototype for an automatic pill reminder.

\section{References}

[1] AARDEX. Medication Event Monitoring System, AARDEX Pill Counter (2005), http://www.aardexgroup.com/aardex_index.php?group=aardex\&id=85 (retrieved 2019 , 2010)

[2] Bernal, I., Domenech, E., Garcia-Planella, E., Marin, L.: Medication-taking behavior in a cohort of patients with inflammatory bowel disease. Digestive Diseases and Sciences 51, 2165-2169 (2006)

[3] Boyle, D., Bubalo, J.: Enhancing Patient Adherence to Improve Outcomes With Oral Chemotherapy, US Pharmacist (2007), http://www.uspharmacist.com (retrieved 10 20, 2010)

[4] Chen, G., Yan, B., Shin, M., Kotz, D.: MPCS: Mobile-Phone Based Patient Compliance. Mobile and Ubiquitous Systems: Networking \& Services , 1-7 (2009)

[5] Claxton, A., Cramer, J., Pierce, C.: Systematic review of the associations between dose regimens and medication compliance. Clinical Therapy 23, 1296-1310 (2001)

[6] Dinwiddie, R., Muller, W.: Adolescent treatment compliance in asthma. JRSM 95, 68-71 (2002)

[7] Dolan, B.: Wireless health currently a $\$ 304 \mathrm{M}$ market., from Mobihealth News (2009), http://mobihealthnews.com/5722/ctia-wireless-health-currently-a-304m-market/\#more5722 (retrieved 1022,2010 )

[8] Donovan, J.: Patient decision making: The missing ingredient in compliance. IJTAHC 11, 443-455 (1995)

[9] Donovan, J., Blake, D.: Patient non-compliance: Deviance or reasoned decision? SSM 34, 507-513 (1992)

[10] Fielding, D., Duff, A.: Compliance with treatment protocols: Interventions for children with chronic illness. Archives of Disease in Childhood 80, 196-200 (1999) 
[11] Gagnon, J., Grenier, R.: Evaluation and validation of quality care indicators relative to empowerment in complex chronic disease. l'Universite Laval, Faculte des Sciences Infirmieres. Quebec (2004)

[12] Jackevicius, C., Mamdani, M., Tu, J.: Adherence with statin therapy in elderly patients with and without acute coronary syndromes. JAMA 288, 462-467 (2002)

[13] Mieczkowski, T.: Drug testing technology: Assessment of field applications. CRC Press, Boca Raton (1999)

[14] Morris, L., Schulz, R.: Patient compliance: An overview. J. Clini. Pharm. and Therap. 17, 183-195 (1992)

[15] Osterberg, L., Blaschke, T.: Adherence to medication. The New England J. of Med. 353, 487-497 (2005)

[16] Ownby, R.L.: Development of an Interactive Tailored Information Application to Improve Patient Medication Adherence. In: AMIA Annu. Symp. Proc., p. 1069 (2005)

[17] Rapoff, M., Barnard, M.: Compliance with pediatric medical regimens. In: Cramer, J., Spiker, B. (eds.) Patient Compliance in Medical Practice and Clinical Trials, pp. 73-98. Raven, New York (1991)

[18] Sackett, D., Snow, J.: The magnitude of adherence and nonadherence. In: Haynes, R., Taylor, D., Sackett, D. (eds.) Compliance in Health Care, pp. 11-12. Johns Hopkins University Press, Baltimore (1979)

[19] Silva, J., Mouttham, A., Saddik, A.E.: UbiMeds: A mobile application to improve accessibility and support medication adherence. In: ACM Proceedings, pp. 71-78 (2009)

[20] Tebbi, C.: Treatment compliance in childhood and adolescence. Cancer Suppl. 71(10), 3441-3449 (1992)

[21] Vermeire, E., Hearnshaw, H., Van Royen, P., Denekens, J.: Patient adherence to treatment: three decades of research - a comprehensive review. J. of Clinical Pharmacy and Therapeutics 26, 331-342 (2001)

[22] Winnick, S., Lucas, D., Hartman, A.: How do you improve compliance? JAAP 115, 718-724 (2005)

[23] World Health Organisation, Adherence to long-term therapies. WHO (2003) 